\title{
EFFECT OF SIALIDASE ON BLOOD GROUP SPECIFICITY OF HOG SUBMAXILLARY GLYCOPROTEINS*
}

\author{
David AMINOFF and Marianne P.MORROW \\ Departments of Internal Medicine (Simpson Memorial Institute) \\ and Biological Chemistry, The University of Michigan, \\ Ann Arbor, Michigan 48104, U.S.A.
}

Received 6 May 1970

Revised version received 26 May 1970

\begin{abstract}
Hogs can be divided into three phenotypes A, H and In, depending on the ability of extracts of their submaxillary glands to inhibit the agglutination of human $A$ cells with human anti- $A$, and human $O$ cells with eel-anti-H serum. Evidence is here provided that the inactive glycoprotein, In, represents $\mathrm{H}$ active glycoprotein containing additional $N$-glycolyl neuraminic acid, which stereospecifically masks the $\mathrm{H}$ determinant, $\alpha(1 \rightarrow 2)$-L-fucosyl residue.
\end{abstract}

\section{Introduction}

It had previously been reparted that hogs can be subdivided into three principal groups: $\mathbf{A}, \mathrm{H}$ or In, depending on their cross-reactivity with human anti-A, or eel anti-H serum, where In represents the absence of $A$ or $H$ reactivity [1]. While the distinction between $\mathrm{A}+$ and $\mathrm{A}-$ is quite sharp, the distinction between $\mathrm{H}+$ and $\mathrm{H}-$, or In, is not as clear-cut. Indeed in many respects, the non $\mathrm{A}$-active, $\mathrm{H}+$ and $\mathrm{H}-$ glycoproteins behave very similarly and the distinction could only be made on the basis of the use of eel anti-H serum.

In the course of purification, it became apparent that the sialic acid content of the In glycoprotein was higher than in the $\mathrm{A}$ and $\mathrm{H}$ glycoproteins. The phossibility arose that the apparent lack of $\mathrm{A}$ or $\mathrm{H}$ activity could be attributable to the masking of the appropriate serologically active sites by sialic acid residues. Previous work indicated that the removal of the sialic acid by mild acid hydrolysis resulted in no change in serological specificity and we were led to conclude

\footnotetext{
* Part IV in the series 'Enzymes That Destroy Blood Group Specificity'. Part III, K.Furukawa and D.Aminoff, in: Blood and Tissue Antigens, ed. D.Aminoff (Academic Press, New York, 1970) pp. 415-426, "Action of Glycosidases on Erythrocytes".
}

that sialic acid did not play such a masking role [1].

Re-investigation of the problem with the use of sialidase indicated that $\mathrm{H}-$ glycoprotein would now react with eel anti-H. The inability to show this after acid hydrolysis is attributable to the simultaneous release of $\alpha(1 \rightarrow 2)$-L-fucosyl residues, the $\mathrm{H}$ determinant, during the acid hydrolysis.

\section{Materials and methods}

Fresh submaxillary glands were obtained from individual hogs from a local slaughter house and processed separately. The glands were chilled in ice immediately on excision. Fat and connective tissue removed, the glands were cut into small pieces and blended with three volumes of water, centrifuged free of residues and the supernatant tested for its serological activity. Typical results for analysis of individual glands are shown in table 1. Analysis of over a hundred glands gave the distribution shown ir fig. 1. Extensive purification of the glycoproteins in the aqueous extracts by modification of procedures described elsewhere $[2,3]$ gave products with the following analytical values and ratios (table 2 ).

The total protein content was determined by the 
Table 1

Determination of the phenotype of individual hogs.

\begin{tabular}{lrrl}
\hline Hog & \multicolumn{2}{l}{ Inhibition titer } & \\
\cline { 2 - 4 } & A & H & \\
\hline 20 & 4 & 16 & In \\
26 & 4096 & 4 & A \\
27 & 64 & 16 & In (A) \\
29 & 4 & 64 & In (H) \\
38 & 4 & 4096 & H \\
\hline
\end{tabular}

microbiuret procedure [4]. Total galactose and fucose were determined by the anthrone method [5], under conditions where both sugars give equimolar extinction coefficients and the contribution due to fucose was determined directly [6]. Total sialic acid was assayed by a modified resorcinol method [7], where $N$-glycolylneuraminic acid (NGN) gives $134 \%$ of the intensity of color given by $\mathrm{N}$-acetylneuraminic acid (NAN). Sugars released by acid or enzymatic hydrolysis were determined by the thiobarbituric acid proce.

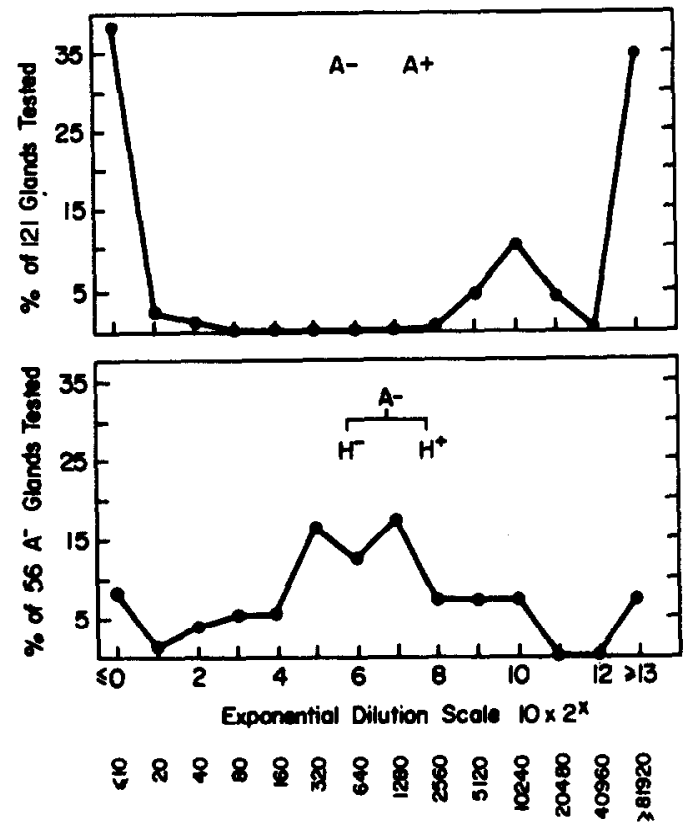

Fig. 1. Distribution of blood group $A$ and $\mathrm{H}$ activities in hog submaxillary glands. dure [8] for sialic acid and the Conway unit procedure for free fucose [9]. $N$-Glycolyl neuramic acid gives $77 \%$ of the intensity of color given by NAN in the thiobarbituric acid assay.

The following reagents were used for the serological test: a) human anti-A, b) human anti-B, c) human anti-Le $e^{\mathrm{a}}, \mathrm{d}$ ) natural eel (Anguilla anguilla) serum for anti-H [10] , e) Ulex europeus anti-H [11], and f) chicken anti-H reagent prepared by injecting chickens with purified hog-H submaxillary glycoprotein (we are grateful to Dr. Ken Furukawa for the anti-H chicken serum). The serological activities were determined by the inhibition of hemagglutination titration method [3].

Acid hydrolysis with $0.1 \mathrm{~N} \mathrm{HCl}$ at $100^{\circ}$ and 79 and $0.5 \mathrm{~N} \mathrm{HCl}$ at $60^{\circ}$ was carried out in sealed ampules in constant temperature baths. The course of hydrolysis was followed by the thiobarbituric acid test for sialic acid and Conway unit assay for free fucose released. The results are shown in fig. 2 .

Enzymatic hydrolysis was carried out with a preparation of silidase isolated from Clostridium perfringens by a modification of the published procedure [12] . The incubation mixture contained approximately $0.25 \mu \mathrm{m}$ of bound NGN, $2 \mu \mathrm{m}$ of tris maleate $\mathrm{pH}$ 5.8 and 0.025 units of sialidase in a total volume of $100 \mu 1$. The rate of release of sialic acid was determined by the thiobarbituric acid procedure.

\section{Results}

The determination of the phenotype of the hogs on the basis of inhibition titer of aqueous extracts of individual submaxillary glands is shown in table 1. Fig. 1 shows the distribution of activity of the submaxillary gland extracts for A+ and A- phenotypes and of the $\mathbf{A}$ - phenotypes on the basis of their reactivity with eel anti-H serum as $\mathrm{H}+$ and $\mathrm{H}-$. The extracts reacting as $\mathrm{A}-\mathrm{H}-$ would be classified as Inactive or In. This distinction between $\mathrm{H}+$ and $\mathrm{In}$ is by no means detectable by the use of other anti- $\mathrm{H}$ reagents as shown in table 3 for the reactivity of purified glycoproteins. It will be noted that only the $A$ active glycoproteins show any inhibiting activity in the Forss. man system [3]. Lea activity could not be demonstrated in submaxillary glycoproteins from hogs of A, $\mathrm{H}$, and In phenotypes. Likewise, none of the hog 
Table 2

Analy tical ratios of purified hog submaxillary glycoproteins.

\begin{tabular}{clllll}
\hline & & & \multicolumn{3}{c}{ Carbohydrate composition } \\
\cline { 5 - 6 } Phenotype & Protein $\%$ & Galactose & Fucose & $\begin{array}{l}N \text {-Glycolyl- } \\
\text { neuraminic } \\
\text { acid }\end{array}$ & $\begin{array}{l}N \text {-Acetyl- } \\
\text { galactosamine }\end{array}$ \\
\hline A & 34.5 & 0.95 & 1 & 0.81 & 1.80 \\
A & 34 & 0.90 & 1 & 0.85 & 1.83 \\
H & 32.4 & 1.11 & 1 & 0.84 & 1.32 \\
In & 38 & 0.92 & 1 & 1.36 & 1.16 \\
In & 41 & 0.84 & 1 & 1.14 & - \\
\hline
\end{tabular}

submaxillary gland extracts tested showed any B activity.

The carbohydrate composition of the purified glycoproteins obtained from pools of submaxillary glands of the same phenotype are shown in table 2 . The two A preparations represent two different pools of glands prepared by slightly different methods and indicate the reproducibility of the data. The higher sialic acid content of the purified In glycoprotein is readily evident. The fact it does not represent a whole integer and, moreover, that preparations obtained from different pools of In glands give different sialic

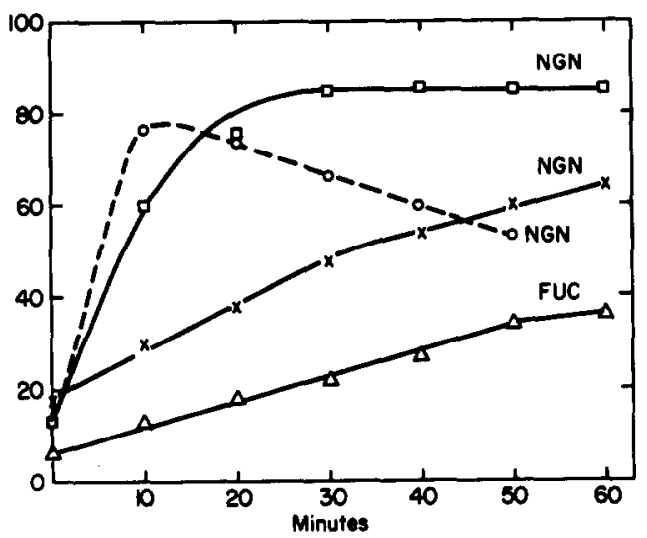

Fig. 2. Rate of release of $N$-glycolyl neuraminic acid (NGN) and fucose on acid hydrolysis of hog-H submaxillary glycoprotein. $\odot-0.1 \mathrm{~N} \mathrm{HCl}$ at $100^{\circ} ; \square \longrightarrow 0,0.1 \mathrm{~N} \mathrm{HCl}$ at $79^{\circ}$; $x-x, 0.5 \mathrm{~N} \mathrm{HCl}$ at $60^{\circ} ; \triangle \triangle, 0.1 \mathrm{~N} \mathrm{HCl}$ at $79^{\circ}$, fucose. acid content suggested that this was possibly responsible for the inactivity.

Acid hydrolysis of hog.H submaxillary under various conditions is shown in fig. 2. The results indicate that under the optimal conditions of hydrolysis, $0.1 \mathrm{~N} \mathrm{HCl}$ at $79^{\circ}$, to release the maximum of sialic acid without extensive degradation of the sialic acid, there is a simultaneous release of fucose which would explain the lack of detection of $\mathrm{H}$ reactivity after the mild acid hydrolysis of In-glycoproteins.

Hydrolysis of the purified A, $\mathrm{H}$ and In-glycoproteins with a purified and potent preparation of siali: dase from $\mathrm{Cl}$. perfringens (in the ratio of 0.07-0.09 $\mu \mathrm{m}$ of bound NGN to 0.125 units of enzyme) resulted in the release of sialic acid as shown in fig. 3 . The rate of release of NGN is strictly comparable for the $A$ and $\mathrm{H}$ glycoproteins, but appears to be two-phased in the case of the In-glycoprotein. The course of hydrolysis was followed serologically with eel anti-H serum. The results, fig. 4, indicate that both the $\mathrm{H}$ and In-glycoprotein show an initial enhancement of $\mathrm{H}$ reactivity,

Table 3

Serological properties of hog submaxillary glycoproteins.

\begin{tabular}{|c|c|c|c|c|c|}
\hline \multirow{3}{*}{$\begin{array}{l}\text { Pheno- } \\
\text { type }\end{array}$} & \multicolumn{5}{|c|}{ Inhibition titer } \\
\hline & \multicolumn{2}{|r|}{$\mathbf{A}$} & \multicolumn{3}{|c|}{ H } \\
\hline & $\mathbf{A}$ & Forssman & Eel & Ulex & Chicken* \\
\hline A & 8,000 & 8,000 & $<200$ & 51,200 & 25,600 \\
\hline $\mathrm{H}$ & $<200$ & 2 & 3,200 & 307,000 & 800,000 \\
\hline In & $<200$ & 2 & $<200$ & 307,000 & 800,000 \\
\hline
\end{tabular}

* Chicken immunized with hog $\mathrm{H}$ submaxillary glycoprotein. 


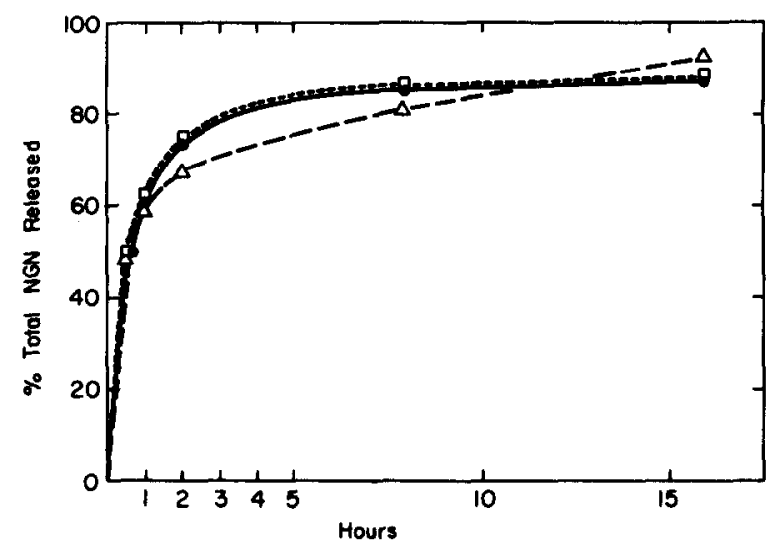

Fig. 3. Rate of hydrolysis of $A, H$ and In hog submaxillary glycoproteins with $C l$. perfringens sialidase, 0.125 units of enzyme, $2 \mu \mathrm{m}$ of pH 5.8 tris maleate buffer in $100 \mu \mathrm{l}$ of incubation mixture containing the following amounts of substrate, expressed as $\mu$ moles of bound $N$-glycolylneuraminic acid, (NGN); $\bullet, A(0.075) ; \square \longrightarrow, H(0.073) ; \triangle \longrightarrow$, In (0.091).

reaching a maximum in $2 \mathrm{hr}$ followed by a slow decline. Chromatographic examination of hydrolysis products of an analogous incubation revealed the presence of NGN and fucose from both the $\mathrm{H}$ and In glycoproteins. The release of fucose with this enzyme under the conditions specified would account for the subsequent loss of $\mathrm{H}$ specificity and indica tes contamination of the enzyme with small amounts of fucosidase which was not detectable in the short in-

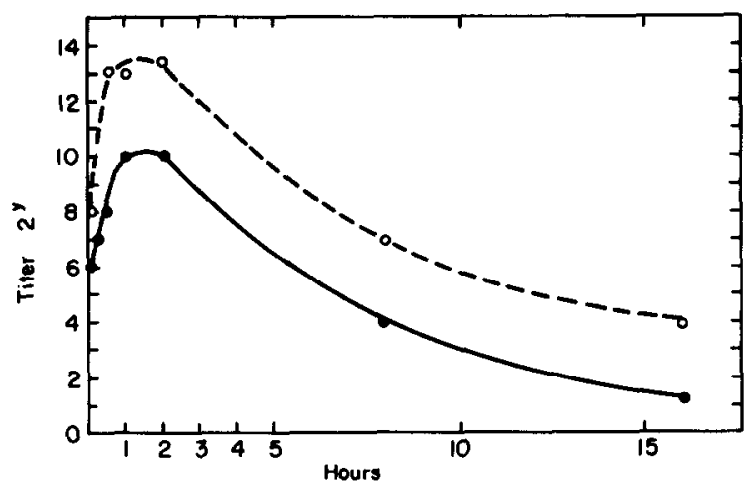

Fig. 4. Changes in $\mathrm{H}$-activity associated with sialidase hydrolysis under the condition described in fig. $3 . \circ-0, \mathrm{H} ; \bullet-$ In.

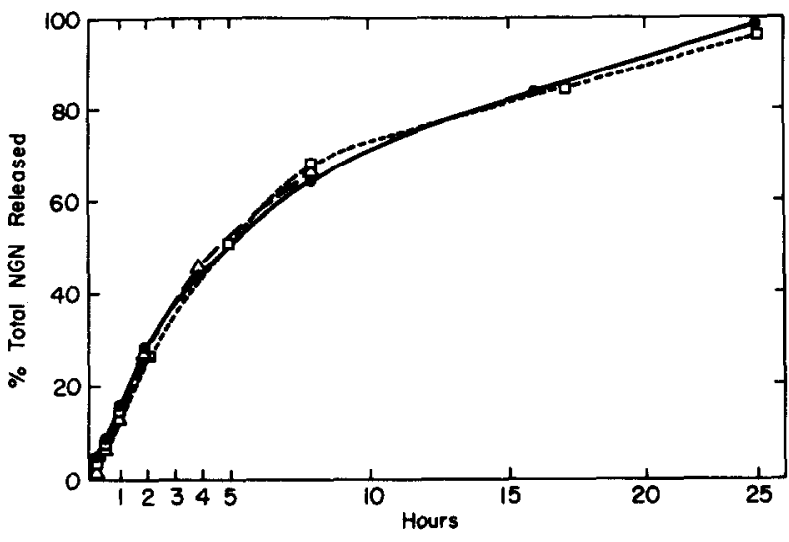

Fig. 5. Rate of hydrolysis of A, H and In hog submaxillary glycoprotein with $\mathrm{Cl}$. perfringens sialidase, 0.025 units, 2 $\mu \mathrm{m}$ of $\mathrm{pH} 5.8$ tris maleate buffer in $100 \mu$ l of incubation mix ture containing the following amount of substrate, expressed as $\mu$ moles of bound NGN, $\longrightarrow$, A (0.23); $\square-\infty$, $H(0.252) ; \triangle \longrightarrow$, In (0.221).

cubation assays, but evident after prolonged incubations.

Repeating the incubations with sialidase under substrate saturation conditions, as indicated in fig. 5 , rather than in the presence of excess sialidase (figs. 3

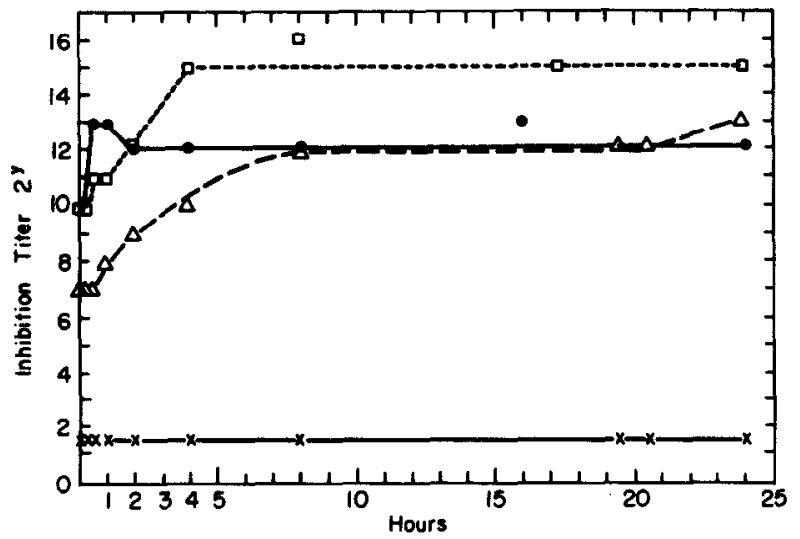

Fig. 6. Changes in $\mathrm{A}$ and $\mathrm{H}$-activity associated with sialidase hydrolysis under the conditions described in fig. 5. $\longrightarrow$, A-activity of A glycoprotein; $\square \longrightarrow, \mathrm{H}$-activity of $\mathrm{H}$-glycoprotein; $\mathbf{x}-\mathbf{x}, \mathbf{A}$-activity of In glycoprotein; $\stackrel{\sim}{\sim}$ H-activity of In glycoprotein. 
and 4) resulted in a slower release of the total sialic acid extending over $20 \mathrm{hr}$ when all the NGN was released. The rate of release from the $\mathrm{A}, \mathrm{H}$ and Inglycoproteins was comparable, with no indications of a two-phased reaction for In-glycoprotein. The corresponding serological changes resulting with the extended periods of incubation are shown in fig. 6 and demonstrate that there is no significant effect on the A activity of the A-glycoprotein. There is no development of A acitivity from the In-glycoprotein. Both the $\mathrm{H}$ and In-glycoprotein show a rise in $\mathrm{H}$ reactivity with the eel anti-H. In contrast to the previous experiment where we had excess sialidase and presumably also fucosidase, the $\mathrm{H}$ reactivity persists here; the $\alpha(1 \rightarrow 2)$-L-fucosidase is too weak to be effective.

\section{Discussion}

The Inactive hog submaxillary glycoprotein has been an enigma [13] since its detection [1]. Three phentotypes $\mathrm{A}, \mathrm{O}$ and $i$, where $i$ corresponds to the absence of $A$ or $O$ on the red cells of hogs, had been previously described by Spraque [14]. This was confirmed by Saison and Ingram [15], who further investigated the genetics involved and suggested that the $i$ was the product of a recessive suppressor gene at another locus. The detection of a similar phenomenon in the hog submaxillary glands [1] immediately suggested the analogous secretor, non-secretor relationship in man and the possible identity of the In factor with the Le material. No Le cross-reactivity was detected and, moreover, collaborative work with Dr. Ruth Saison [16] indicated that the two systems, $i i$ on red cells and In in submaxillary glands, were not the same.

Further work to elucidate the nature of the Inglycoprotein was, therefore, called for. The preliminary dat a clearly indicated its closer resemblance to the $\mathrm{H}$ rather than the $\mathrm{A}$ active glycoprotein. Most anti-H reagents do not distinguish between the $\mathrm{H}$ and In-glycoproteins; only eel anti-H serum distinguishes between them (table 2). But even with this reagent there is a variable but persistent trace of $\mathrm{H}$ activity. Moreover, like $\mathrm{H}$ and unlike the $\mathrm{A}$ active glycoproteins, the In glycoprotein shows no Forssman cross-reactivity. Treatment with $\alpha(1 \rightarrow 2)$-L-fucosidase from $\mathrm{Cl}$. perfringens [13] further underscored the resemblance of In to the $\mathrm{H}$ active glycoprotein.
The involvement of sialic acid became evident from the analytical ratios of the sugar components of the purified glycoproteins (table 2). The greater and more variable sialic acid content of the In-glycoprotein (table 2) was suggestive. Treatment with sialidase confirmed the suspicion. Contrary to the general title of this series of investigations, sialidase appears to enhance rather than destroy the blood group activity of the glycoprotein. Thus, the removal of NGN from the oligusaccharide chains appears to enhance $\mathrm{H}$ activity, presumably by making the $\mathrm{H}$ determinant, $\alpha(1 \rightarrow 2)$-L-fucosyl residues, more readily accessible to the eel anti-H serum.

The higher molar ratio of NGN:Fucose in In as compared to the $\mathrm{H}$ active glycoprotein, where the two sugars are present in equimolar proportions, would suggest that there is a second NGN residue in the In glycoproteins which further masks its $\mathrm{H}$ reactivity. Evidence for two forms of NGN present in the Inglycoprotein is indicated by the rate of release of NGN (fig. 3).

Removal of NGN from the In-glycoprotein results in the development of $\mathrm{H}$ specificity and never in $\mathrm{A}$ reactivity (fig. 6). This would suggest that the second NGN residue in the In-glycoprotein occupies the same position as the $N$-acetylgalactosamine in the A-active glycoprotein, viz $\alpha(1 \rightarrow 3)$ to galactose [17], and this proximity to the $\alpha(1 \rightarrow 2)$ fucosyl residue attached to the same galactose residue would explain the masking of the $\mathrm{H}$ specificity. Work is in progress to establish this.

\section{Acknowledgements}

This work was supported by Grant AM 07305 from the National Institutes of Health and in part by a grant from The University of Michigan Cancer Research Institute.

\section{References}

[1] D.Aminoff, M.P.Morrow and C.J.D.Zarafonetis, Federation Proc. 23 (1964) 274.

[2] W.T.J.Morgan and H.K.King, Biochem. J. 37 (1943) 640.

[3] D.Aminoff, W.T.J.Morgan and W.M.Watkins, Biochem. J. 46 (1950) 426. 
[4] O.H.Lowry, N.J.Rosebrough, A.L.Farr and R.J.Randall, J. Biol. Chem. 193 (1951) 265.

[5] R.Dreywood, Ind. Eng. Chem. Anal. Edition 18 (1946) 499.

[6] Z.Dische and L.B.Shettles, J. Biol. Chem. 175 (1948) 595.

[7] L.Svennerholm, Biochim. Biophys. Acta 24 (1957) 604.

[8] D.Aminoff, Biochem. J. 81 (1961) 384.

[9] A.K.Bhattacharyya and D.Aminoff, Anal. Biochem. 14 (1966) 278.

[10] B.Jonsson, Acta Pathol. Microbiol. Scand. Suppl. 54 (1944) 456 .
[11] W.C.Boyd and E.Shapleigh, J. Lab. Clin. Med. 44 (1954) 235; Blood 9 (1954) 1195.

[12] J.T.Cassidy, G.W.Jourdian and S.Roseman, J. Biol. Chem. 240 (1965) 3501.

[13] D.Aminoff and Ken Furukawa, in: International Symposium on Blood and Tissue Antigens, ed. D.Aminoff (Academic Press, New York, 1970) p. 395.

[14] L.M.Sprague, Genetics 43 (1958) 906.

[15] R.Saison and D.G.Ingram, Ann. N. Y. Acad. Sci. 97 (1962) 226.

[16] Unpublished observations.

[17] D.M.Carlson, J. Biol. Chem. 243 (1968) 616. 